\title{
Angiogram Negative Subarachnoid Hemorrhage in the Settling of Sexual Intercourse and Chronis Cannabis Use
}

\author{
Dakota May, MS', Dominika Lozowska, MD¹
}

ABSTRACT

Etiology of unprovoked subarachnoid hemorrhage (SAH) is predominantly from cerebral aneurysm rupture and manifests classically as a thunderclap headache. Orgasmic cephalgia may herald SAH given that 4-12\% of SAH sufferers were found to have engaged in prior sexual activity. 1 Precipitating causes of SAH leading to aneurysmal rupture may be the rise in blood pressure caused by physical activity. A CT angiogram (CTA) is used to reveal a source of the bleed but occasionally this is normal, and is labelled angiogram- negative $\mathrm{SAH}$ or non-aneurysmal $\mathrm{SAH}$. In those cases digital subtraction imaging (DSA, conventional angiogram) is needed for verification. Herein we discuss an instance of angiogram negative $\mathrm{SAH}$ which occurred after sexual activity in a young male with a chronic cannabis habit.

\section{KEYWORDS}

Subarachnoid Hemorrhage, Non-Aneurysmal SAH, Marijuana use, Cannabis habit, Post-Coital Headache
Author affiliations are listed at the end of this article.

Correspondence to:

Dakota May, MS

Marshall University

Joan C. Edwards

School of Medicine may110@marshall.edu
A 26-year-old African American male with no significant past medical history presented with complaints of a severe occipital headache that started during sexual intercourse and was non-radiating. His mother passed away from stroke in her thirties and father's medical history was unknown. His siblings had no medical problems. He had used his usual dose of marijuana daily for the last 3 years and smoked 3-4 cigarettes per day. He was allergic to iodine and was not on any medications. He had no intake of alcohol or any toxin exposures. Patient described the headache as throbbing and graded it as $8 / 10$ in intensity. He denied nausea, vomiting, visual disturbance, chest pain, palpitations, numbness, tingling, weakness or gait difficulties. He was afebrile, heart rate was 58, blood pressure 117/68. His neurologic exam was normal, including cranial nerves, strength reflexes, coordination, sensation, gait and station. CT head (Figure 1) showed an acute subarachnoid hemorrhage within basilar cisterns and right temporal lobe along the sylvian fissure with no hydrocephalus. Neurology recommended
BP goal $<140 / 90$ and admission to ICU for further management. He was started on nimodipine $60 \mathrm{mg}$ q4h for 21 days (7 of which he spent as inpatient). Laboratory testing showed a normal white blood cell count, electrolytes, liver functions, and urine drug screen showed presence of cannabinoids. Patient's headache went down to 3/10 intensity in the first 12 hours and after that he was asymptomatic. CTA head (Figure 2) showed no evidence of arteriovenous malformation (AVM) or arterio-venous (AV) fistula or intracranial aneurysm. DSA showed that there was mild hypervascularity in the right temporal region, possibly from an AVM. Dural venous sinuses were patent throughout. No aneurysm was identified. MRI brain without contrast (Figure 3) showed no clear source for the observed subarachnoid hemorrhage and the brain appeared normal. Transcranial dopplers performed daily over 7 days were negative for any significant vasospasm. CTA (Figure 4) after 1 week from onset showed resolution of the $\mathrm{SAH}$ and possible dissection flaps involving the cavernous portions of bilateral internal carotid arteries which 


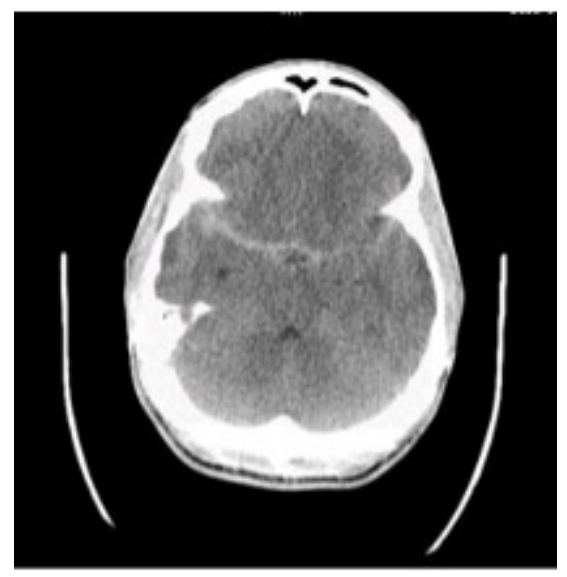

FIGURE 1. CT head showing the SAH upon presentation.

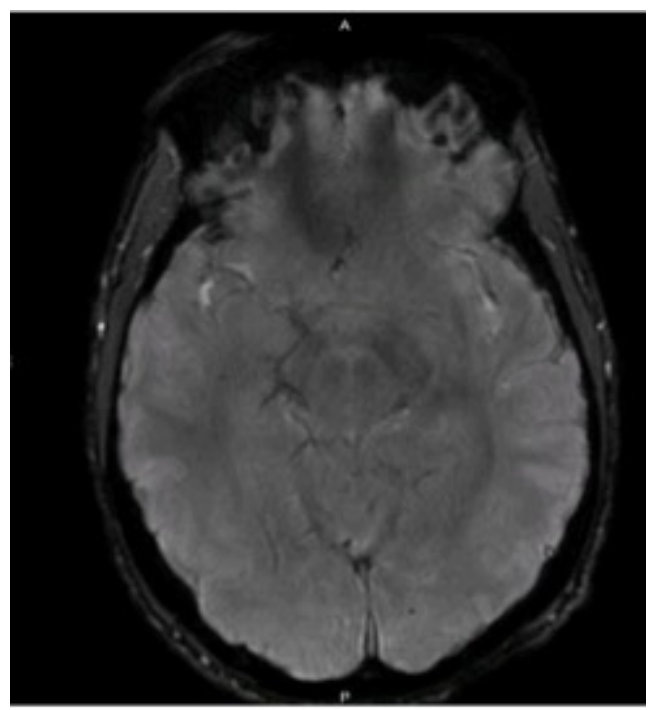

FIGURE 3. A) MRI brain SWAN sequence the day after presentation.
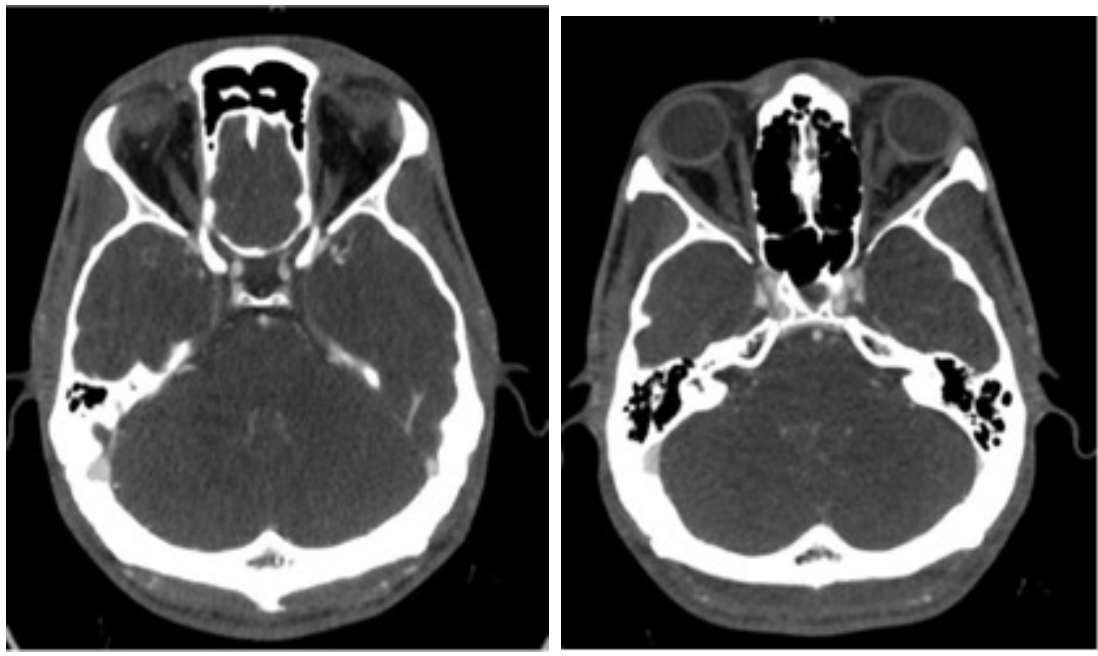

FIGURE 4. CTA performed 1 week after onset of symptoms showed possible dissection flaps involving the cavernous portions of the internal carotid arteries. This may represent bony artifact. 
ultimately were determined to be bony artifact.

\section{DISCUSSION}

Angiogram negative $\mathrm{SAH}$ (or non-aneurysmal SAH) accounts for $15-20 \%$ of all $\mathrm{SAH}^{2}$ and is believed to be caused by: venous drainage anomalies or venous sinus thrombosis; spinal or cerebral dural or parenchymal vascular malformation or tumors in the brain, spine, or subarachnoid space; infections; blood dyscrasias, complications of anticoagulants and fluoxetine, trauma, and small perforating vessel rupture. ${ }^{2}$ Our patient may have had a small vessel rupture that was not picked up on any of the three angiograms that were performed.

As per MacKinnon et al, the incidence of spontaneous $\mathrm{SAH}$ worldwide is approximately 9 per 100,000 persons peaking in the sixth decade. ${ }^{3}$ According to Nayak et al., in their classification system (Table 1), Type $1 \mathrm{a}$ and $1 \mathrm{~b}$ SAH which are localized to basal cisterns and sylvian fissure only, have benign outcomes, based on their study of 129 type $1 \mathrm{SAH}$ patients. ${ }^{2}$ Despite the likelihood that his prognosis would likely be benign, our patient was not discharged but admitted to observation in the hospital because the initial CT imaging raised the possibility of an underlying AVM and also because most cases of vasospasm occur on days 3-7. Once the MRI brain disproved any AVM, DSA was employed to not miss any aneurysm which may not have been noticed, as sometimes CTA misses a small aneurysm due to various reasons such as small aneurysm lumen size, technical factors like poor contrast timing, and large blood burden. ${ }^{4}$ Foreman et al reported finding that 4 out of 16 patients retrospectively studied with post-coital SAH had no brain pathology. ${ }^{4}$

Non-aneurysmal SAH during sexual activity may be due to high blood pressure affecting spiking of mean arterial pressure (MAP) and impacting cerebral autoregulation negatively. Also, intercourse leads to elevated heart rate causing a hyperdynamic state. ${ }^{3}$ Oral erectile dysfunction treatment popular nowadays is with phosphodiesterase- 5 inhibitors and these have been reported to be linked to both aneurysmal and non-aneurysmal SAH, especially during their illicit use, ${ }^{5,6,7,8}$ our patient, however, denied using these medications.

It would be helpful to have information about further relatives of patient in order to determine if there was any possibility of Ehlers-Danlos (EDS) vascular type in the family, however, he was lost to follow up. EDS makes one prone to dissection and possibly hemorrhage. The angiogram did not indicate any trace of Call - Fleming syndrome either, also known as reversible vasoconstriction syndrome (RCVS), or of cerebral vasculitis which may cause acute headache.

Patient's cannabis habit may have been a risk factor because vasoactive substance use may contribute to a subarachnoid hemorrhage. Rumalla et al reported that recreational marijuana use is independently associated with an increased likelihood of $\mathrm{SAH} .{ }^{9}$ The incidence of a SAH among cannabis users was 10.67 times greater than expected when adjusted for age, 13.20 times greater than expected when adjusted for gender, and 8.71 times greater than expected when adjusted for race. ${ }^{9}$ This fact is not widely known, and further studies are needed.

The main psychoactive component of cannabis (tetrahydrocannbinol) effects mitochondria and oxidative stress, while cannabis cessation showed resolution of multifocal intracranial stenosis in $21 \%$ of young patients in a series with stroke. ${ }^{10}$ Transcranial doppler (TCD) studies have shown elevated cerebrovascular resistance in cannabis users, which may increase susceptibililty to delayed cerebral ischemia, along with elevated systolic velocities. ${ }^{10}$ Curiously our patient did not have concerning TCD results. He did not have any striking structural differences on vascular imaging (Figure 2, Figure 4) so there may still be an unknown component that contributed to his presentation and worked in combination with his vasoactive substance use, with the rapid increase in blood pressure and heart rate which occur in intercourse, to cause his angiogram- negative $\mathrm{SAH}$.

The take away points and conclusions that come to mind with this case are that given that Rumalla et al saw a risk of an $18 \%$ increase for SAH correlating to cannabis, which is not insignificant, caution should be exercised when using vasoactive substances in the setting of activities that already act to increase heart rate and blood pressure naturally, as does 


\begin{tabular}{|c|c|}
\hline Subarachnoid Hemorrhage Types 1-4 & Distribution \\
\hline Type 1A & Blood in Basal Cisterns only \\
\hline Type 1B & $\begin{array}{c}\text { Blood in Basal Cisterns w/ Extension into } \\
\text { the Sylvian Fissures }\end{array}$ \\
\hline Type 2 & $\begin{array}{c}\text { Type 1 (1a \& 1b) w/ Extension of Blood } \\
\text { into Cortical Sulci Ventricles }\end{array}$ \\
\hline Type 3 & SAH w/out Blood in Basal Cisterns \\
\hline Type 4 & $\begin{array}{c}\text { Any Type of SAH with Subarachnoid blood in } \\
\text { the Posterior Fossa/Cerebellar Vermis }\end{array}$ \\
\hline
\end{tabular}

TABLE 1. Illustration of the SAH classification categories from Nayak et al. ${ }^{1}$

intercourse. So perhaps when taking social history physicians could consider counselling marijuana users to take care, or at least be informed about their risk for rare but potentially grave neurologic consequences, the mechanism of which is not fully understood. With legalization of medical and in some states recreational marijuana, it is useful to present this case for discussion. Certain corollaries exist; for example, valsalva manouvers have occasionally been linked to aneurysmal rupture and straining when constipated may potentially increase intracranial pressure, but there are many factors at work as to why sometimes a serious complication results. Similarly, it is conceivable that blood redistribution and cerebrovascular resistance may play a role in causing $\mathrm{SAH}$ when vasovagal substances like marijuana are used but there may be other understudied or unknown predictors.

The rationale for these conclusions is that while this patient had no long lasting repercussions, it was still a serious encounter that could have had dire consequences. He was an otherwise normal young male with the only risk factor of stroke being smoking. It could have turned out quite differently for another patient in a similar situation who had more vascular risk factors, or at another time for this same patient if he combined another vasovagal drug with the marijuana. Essentially, further study is needed on this subject, preferably as a case series. Cannabis has had a long history of use in the world, so it may be useful to keep track of similar cases of non-aneurysmal post-coital SAH coinciding with marijuana use, if they can be amassed, although they may be rather under-reported due to the sensitive and divisive nature of the topic.

\section{AUTHOR AFFILIATIONS}

1. Marshall University Joan C. Edwards School of Medicine, Huntington, West Virginia

\section{REFERENCES}

1. Singh $D$, Jan A. Subarachnoid haemorrhage with orgasmic cephalgia. BMJ Case Rep. 2013;1-2.

2. Nayak S, Kunz AB, Kieslinger K, Ladurner G, Killer M. Classification of non-aneurysmal subarachnoid haemorrhage: CT correlation to the clinical outcome. Clin Radiol. 2010;65(8):6238.

3. Mackinnon AD, Clifton AG, Rich PM. Acute subarachnoid haemorrhage: Is a negative CT angiogram enough? Clin Radiol. 2013;68(3):2328.

4. Foreman PM, Griessenauer CJ, Selim MH, Searls DEC, Safdar A, Kasper EM, et al. Sexual activity as a trigger for intracranial hemorrhage. Acta Neurochirurgica. 2016;189-95.

5. Kaneria MV, Pagar S, Samant H, Yeole S, Patil S. Subarachnoid haemorrhage: possibly caused by the illegitimate use of sildenafil citrate. Journal 
Assoc Physicians India. 2006;56:809-811.

6. Byoun HS, Lee YJ, Yi HJ. Subarachnoid hemorrhage and intracerebral hematoma due to sildenafil ingestion in a young adult. Journal of Korean Neurosurgery Society. 201;47(3):210-212.

7. DeGiorgio F, Arena V, Arena E, Lodise M, Valerio L, d'Aloja E, Chiarott M. Subarachnoid hemorrhage during sexual activity after sildenafil intake: an accidental association. American Journal Forensic Med Pathology. 2011;23(4)310-311.

8. Antar V, Sutpideler N, Baran O, Bitirak G. Subarachnoid and intracerebral hemorrhage after alcohol ingestion and illicit use of sildenafil. Turkish Neurosurgery. 2015;25(3):485- 7.

9. Rumalla K, Reddy AY, Mittal MK. Association of recreational marijuana use with aneurysmal subarachnoid hemorrhage. J Stroke Cerebrovasc Dis. 2016;25(2):452-60.

10. Behrou R, Birnbaum L, Grandhi R, Johnson J, Misra V, Palacio $S$ et al. Cannabis use and outcomes in patients with aneurysmal subarachnoid hemorrhage. Stroke. 2016;13711373. 